Eduardo F. Marques Ali Khan

\section{Effect of a bile salt on the aggregation behavior of a double-chained cationic surfactant - the cationic-rich dilute region of the didodecyldimethylammonium bromide- sodium taurodeoxycholate-water system}

E. F. Marques $\cdot$ A. Khan $(\bowtie)$

Physical Chemistry 1, Center for Chemistry and Chemical Engineering, P.O. Box 124, Lund University, Lund 22100 , Sweden e-mail: ali.khan@fkem1.lu.se

E. F. Marques $(\bowtie)$

Centro de Investigação em Química,

Department of Chemistry,

Faculty of Science,

University of Porto,

Rua do Campo Alegre 687,

4169-007 Porto, Portugal

e-mail: efmarque@fc.up.pt

\begin{abstract}
The phase behavior and aggregate structure of the didodecyldimethylammonium bromide (DDAB)-sodium taurodeoxycholate-water mixed surfactant system have been investigated in the dilute (above $95 \mathrm{wt} \%$ water) cationic-rich area, at $25^{\circ} \mathrm{C}$. A combination of techniques has been used, including polarizing and video-enhanced light microscopy, cryogenic transmission electron microscopy and water selfdiffusion NMR. Between the dilute lamellar phase $(\approx 4-30 \mathrm{wt} \%$ DDAB $)$ and the very narrow isotropic solution (below $0.5 \mathrm{wt} \%$ DDAB), the lamellar dispersions formed by DDAB contain different types of vesicle structures (vesicles, microtubules and multilamellar structures). On addition of bile salt to the DDAB dispersions, strong electrostatic headgroup interactions and geometric packing effects (owing to the unusual molecular structure of
\end{abstract}

the anionic surfactant) are present. The vesicle aggregates, however, are able to incorporate an amount of bile salt up to roughly $20 \mathrm{~mol} \%$. Further addition induces a macroscopic phase separation, with the formation of a strong white dispersion. The light and electron micrographs show that the vesicles are spherical and integral, undergoing an increase in size when the bile salt concentration is varied between 0 and $10 \mathrm{~mol} \%$. At higher bile salt concentration, smaller vesicles, tubular structures and submicronsized dispersion droplets are observed. Water self-diffusion NMR measurements give further information regarding vesicle size and polydispersity.

Key words Surfactant phase behaviour - Catanionic mixtures . Bile salts $\cdot$ Double-chained surfactants $\cdot$ Vesicles

\section{Introduction}

The bile acid salts are a vital class of biological surfactants, as they are directly linked to the mechanism of emulsification and absorption of lipids in the human digestive tract $[1,2]$. They act in the body mixed with cholesterol and lecithin in the form of mixed micelles. The three components form the complex biological mixture known as bile, in which various types of selfassembled aggregates (micelles and vesicles) have been imaged by microscopy techniques [3]. The bile salts are also believed to have a central role in the process of excretion of cholesterol and lecithin from the body. In connection with the molecular understanding of these processes, several studies have been performed on the aqueous phase behavior of mixtures of bile salts with lecithin $[4,5,6,7,8]$, with lecithin and cholesterol [9] and with monoglycerides [10]. In particular, in dilute systems the structural characterization of the disintegration process of phospholipid vesicles upon addition of bile 
salts has received continuous attention in connection to its biological relevance $[5,7,8,11,12]$.

Studies on mixed systems of bile salts with nonnatural surfactants have also been reported, in particular with some single-chained surfactants, both at high and low total amphiphile concentrations [13, 14]; however, the interactions between a bile salt and an oppositely charged double-chained amphiphile have not been addressed to the same extent. These mixed systems composed of cationic and anionic surfactants have been generally termed catanionic mixtures and their properties have been extensively investigated for decades $[15,16,17,18]$. In recent years particular emphasis has been given to phase behavior properties in dilute $[19,20,21,22,23,24,25]$ and concentrated systems $[26,27,28]$. In this work, the phase behavior and aggregate structure for the sodium taurodeoxycholate (STDC)-didodecyldimethylammonium bromide (DDAB) system, in the dilute DDAB-rich area, is presented. This region is dominated by relatively complex equilibria between different bilayer aggregates. The DDAB molecule has low solubility in water and its packing parameter, close to unity, dictates a preference to assemble into bilayer-based structures. At room temperature, DDAB in water displays the formation of two lamellar liquid-crystalline phases [29, 30, 31, 32], with an upper critical point, and, at high dilution, complex equilibria involving giant multilamellar vesicles (onions) and smaller unilamellar and bilamellar vesicles $[25,33,34,35,36]$.

The DDAB-bile salt catanionic pair investigated here has clear similarity with the lecithin-bile salt pair, which has been extensively investigated. Both the phospholipid and DDAB are vesicle- and lamellarforming amphiphiles; however, the presence of the headgroup electrostatic interactions and salt $(\mathrm{NaBr})$ in the catanionic system brings a new complexity to the phase equilibria and aggregation processes. Previously, the phase behavior and aggregation phenomena were studied for the same catanionic pair, in the bile salt rich area [37]. It was reported that the system displays coacervation instead of precipitation at equimolarity, consisting of a viscous isotropic solution in equilibrium with a very dilute solution. In the water-rich part of the phase diagram, a peculiar type of phase separation occurs, involving the formation of very fine bluish dispersions where large micellar-like domains were imaged by electron microscopy. As discussed later, a similar type of fine dispersions occurs on the DDABrich side; however, the region close to the binary DDAB-water axis is clearly dominated by bilayer aggregates which are capable of accommodating the bile salt in their structure up to roughly a $4: 1$ DDAB:STDC molecular ratio.

\section{Experimental}

Materials

STDC, $0.5 \mathrm{~mol} \mathrm{H}_{2} \mathrm{O} / \mathrm{mol}$, more than $97 \%$ in purity, was purchased from Sigma and was used without further purification. DDAB of high purity was obtained from Tokyo Kasei. STDC is the sodium salt of $3 \alpha, 12 \alpha$-dihydroxy- $5 \beta$-taurocholan-24-oic acid. The molecular structure for the two amphiphiles has been shown in a previous report. We only note here that the two $\mathrm{OH}$ groups in the nonplanar steroid ring point to the same side of the ring, in a way that the latter has both a polar and an apolar face.

Sample preparation and phase diagrams

The samples were prepared by weighing directly the two surfactant solids into glass vials and thoroughly mixing them in water endover-end for at least $24 \mathrm{~h}$. The composition of samples is given by weight percent or molar concentration and the mixing ratio of the surfactants is expressed by the molar fraction of bile salt in the mixture, $X_{\mathrm{STDC}}=n_{\mathrm{STDC}} /\left(n_{\mathrm{STDC}}+n_{\mathrm{DDAB}}\right)$, where $n_{i}$ is the number of moles of component $i$ in the sample.

After proper mixing, the samples were inspected over the course of several weeks until equilibration was ascertained. Preliminary studies of the phase behavior involved the observation of samples between crossed polarizers in order to check for birefringence. Further assignment of the phase behavior was done on the basis of combined data supplied by polarizing microscopy, cryogenic transmission electron microscopy (cryo-TEM) and self-diffusion NMR. The uncertainty of the phase boundaries in Fig. 1a is better than $\pm 0.2 \mathrm{wt} \%$.

\section{Polarized light microscopy}

An Axioplan universal polarizing light microscope (Carl Zeiss), equipped with a high-sensitive SIT $\mathrm{C}$ video-camera system and Argus-20 image processor (Hamamatsu Photonics, Japan), was used. In a light microscope equipped with differential interference contrast (DIC) lenses and a television camera connected to the microscope, both contrast and resolution can be improved in what is termed DIC video-enhanced microscopy.This technique is very suitable to detect the presence of micron-sized bilayer structures (e.g. vesicles and microtubules) under normal light. Also, lamellar dispersions containing large multilamellar vesicles (onions) show domains with a characteristic Maltese cross pattern under polarized light [38].

\section{Cryogenic transmission electron microscopy}

This technique is a particularly suitable technique for the direct visualization of surfactant aggregates ranging in size from about 5-10 $\mathrm{nm}$ to $1 \mu \mathrm{m}$. Vitrified samples were prepared, transferred and imaged according to the usual procedure, described in previous works [24, 25, 37]. A Philips CM120 Bio-Twin microscope was used, especially designed for handling cryogenic samples. This microscope was equipped with a lens system giving maximum contrast and high resolution $(3.4 \AA)$, allowing the digital acquisition of images.

\section{Pulsed-field-gradient NMR self-diffusion}

The pulsed-field-gradient (PFG) technique for measuring selfdiffusion coefficients is a powerful technique for structural and dynamic studies in surfactant systems [39, 40]. The method is based 


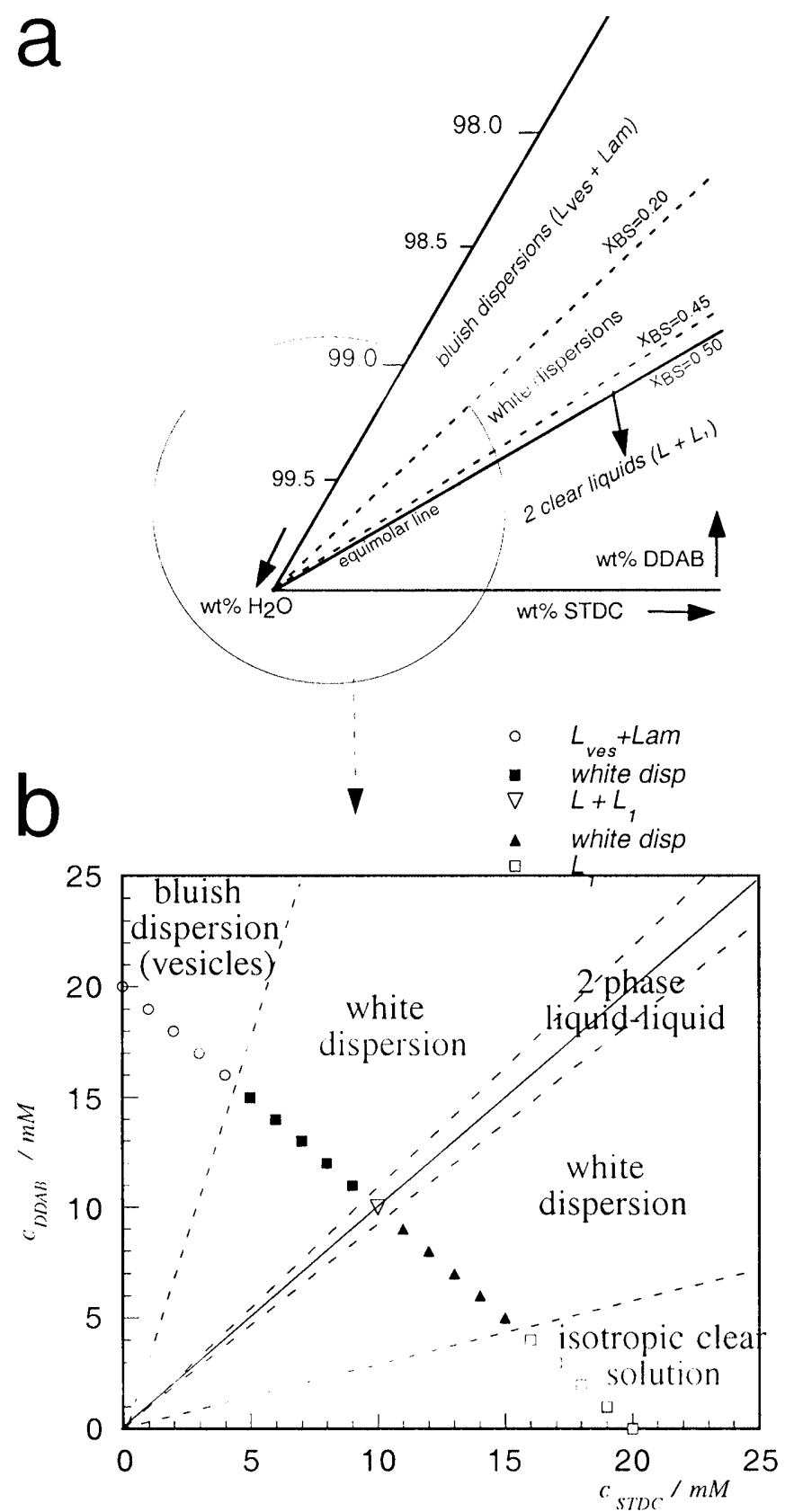

Fig. 1a, b Phase behavior for the dilute region of the didodecyldimethylammonium bromide $(D D A B)$-sodium taurodeoxycholate $(S T D C)$-water system, at $25^{\circ} \mathrm{C}$. a The water apex of the triangular phase diagram (composition on a weight percent basis). b Detailed phase map for the very dilute region obtained from the titration of homogenized dispersions of DDAB and micellar solutions of STDC; the series at constant total surfactant concentration $c_{\mathrm{t}}=20 \mathrm{mM}$ is shown. The full straight line indicates an equimolar composition

on a combination of radio frequency (RF) pulses and magnetic field gradient pulses [41, 42]. The typical PFG sequence is based on the Hahn spin-echo and it consists of two RF pulses, a $90^{\circ}$ and a $180^{\circ}$ pulse, and two gradient pulses placed on either side of the $180^{\circ}$ RF pulse, with a given time duration and magnitude. The diffusion coefficient is determined by measuring the intensity decay of a
Fourier transform starting at the center of the echo - the echo attenuation - as a function of either the duration or the magnitude of the gradient pulse. The attenuation of the spin echo results from the dephasing of the nuclear spins owing both to translational motion of the molecule and to the imposition of the gradient pulse. The measurements were done using a Bruker DMX200 spectrometer, operating at a ${ }^{1} \mathrm{H}$ resonance frequency of $200 \mathrm{MHz}$, with a gradient probe providing a maximum field gradient of $9 \mathrm{~T} / \mathrm{m}$. For the monitoring of the water self-diffusion the basic Hahn echo pulse sequence was used. A detailed description of these pulse sequences can be found in specialist reviews $[40,41]$ or in previous work by us [24].

\section{Results and discussion}

Phase behavior in the dilute region (DDAB $<3 \mathrm{wt} \%$ )

The isothermal phase behavior of the DDAB-STDCwater system can be approximately depicted in a conventional triangular phase diagram, with compositions on a weight percent basis. The DDAB-rich area of the water apex of this triangular phase diagram is shown in Fig. 1a (from combined light microscopy and cryoTEM observations, and water self-diffusion NMR measurements, at $25^{\circ} \mathrm{C}$ ). Also another phase diagram (Fig. 1b) is shown in more detail, for molar concentrations for the very dilute region of the mixture. This phase diagram results from the observation of samples mixed by volume from carefully homogenized dispersions of DDAB and equimolar bile salt micellar solutions to yield a constant total surfactant concentration (in Fig. 1b the series at $c_{\mathrm{t}}=20 \mathrm{mM}$ is shown).

Prior to the description of the phase behavior, it is appropriate to briefly review the aggregation behavior of $\mathrm{DDAB}$ itself in water. The very dilute region of the DDAB-water binary system is very rich in terms of the variety of self-assembled bilayer aggregates $[31,33,34$, $35,36,43]$. The surfactant forms a bluish isotropic solution until at about $0.15 \mathrm{wt} \%(\approx 3.2 \mathrm{mM})$ at $25^{\circ} \mathrm{C}$, where unilamellar vesicles are present (there is also NMR self-diffusion evidence for small micelles [25]). The dilute lamellar phase, $\mathrm{D}_{1}$, which forms between 3 and $30 \mathrm{wt} \%$ $\mathrm{DDAB}$, displays an intense birefringence under polarized light. This phase is built up of micron-sized multilamellar vesicles which occupy all the available volume [34, 35, 44]. In the polarizing light microscope, typical Maltese cross patterns appear in a dark background, indicating the presence of the onion structures [45]. In the intermediate range, $0.15-3 \mathrm{wt} \% \mathrm{DDAB}$, the appropriate two-phase region is present, containing the isotropic solution and the lamellar phase $\mathrm{D}_{1}$. For concentrations between 0.15 and $1.5 \mathrm{wt} \%$, this biphasic region shows itself macroscopically as a bluish pseudo-homogeneous dispersion, commonly designated as a lamellar dispersion. Large unilamellar and bilamellar vesicles with sizes ranging roughly from 100 to $1,000 \mathrm{~nm}$ are present here. When left to stand undisturbed for days, the dispersions 
develop an upper layer containing flocs of giant onions. For the range $1.5-3 \mathrm{wt} \%$, the biphasic region is a very stable dispersion of lamellar $\left(\mathrm{D}_{1}\right)$ "domains" (onions) in the "excess water" phase, a dilute solution containing microtubules and bilamellar vesicles (100-200 nm).

It is shown in Fig. 1 that upon addition of STDC, three distinct regions occur:

1. A bluish dispersion region.

2. A white dispersion region.

3. A two-phase region, with two colorless liquids in coexistence.

The bluish dispersions, present in the DDAB-water system, are able to incorporate an amount of bile salt corresponding roughly to $X_{\mathrm{STDC}}=0.20$. These bluish samples look macroscopically homogeneous and are flow birefringent, showing a gradual loss of birefringence as the bile salt concentration increases. The region for $X_{\mathrm{STDC}}=0.20-0.45$ is entirely different in nature: a white milky-type dispersion is formed, looking very similar to an emulsion. Inside this region, for $X_{\text {STDC }}=0.38-0.45$, it is even possible to see small droplets inside the white dispersion with the naked eye. Finally, the region very close to equimolarity, $X_{\text {STDC }}=0.45-0.5$, is dominated by a coacervate: two colorless liquids are in equilibrium, with the upper one being very viscous. In the bile salt rich area (Fig. 1b) a white milklike dispersion is also formed, between $X_{\text {STDC }}=0.55$ and 0.75 , and for $X_{\text {STDC }}>0.75$ the mixed micellar solution $\left(\mathrm{L}_{1}\right)$ is present, as reported in previous work [37].

Light microscopy and cryo-TEM observations

A representative series of samples in regions 1and 2, at constant $c_{\mathrm{t}}=c_{\text {DDAB }}+c_{\text {STDC }}=20 \mathrm{mM}$, was observed by video-enhanced light microscopy at $25{ }^{\circ} \mathrm{C}$. For all samples in the range $X_{\mathrm{STDC}}=0-0.18$, very large round vesicles in Brownian motion can be seen in the microscope, with size ranges varying between 1 and $40 \mu \mathrm{m}$. This type of structure is illustrated at $X_{\mathrm{STDC}}=0.10$ in Fig. 2a. The vesicles observed are in the range $1-10 \mu \mathrm{m}$, displaying low contrast with the medium, and the overall vesicle fraction seems to be fairly high. The evolution of size upon STDC addition is unclear from the microscopy observations, since the samples are highly concentrated and fairly polydisperse. Ultimately, the bluish dispersion at $X_{\text {STDC }}=0.20$ (Fig. 2b) shows much smaller particles than the ones seen for the dispersions in Fig. 2a. The particles are seen in constant Brownian motion. They appear as spheroidal particles not larger than $2 \mu \mathrm{m}$ and are much less polydisperse than the vesicle aggregates shown in Fig. 2a. The particles which are out of the focus plane are shown as dark spots in Fig. 2a and they appear to consist of small droplets of a phase dispersed in the continuous phase.

When further bile salt is added, small liquid droplets dispersed in a bulk liquid are imaged. This effect can be seen in Fig. 2c, at $X_{\mathrm{STDC}}=0.35$. Similar images are obtained for other samples pertaining to the region where white milky dispersions occur. The droplets are fairly monodisperse (roughly $0.8-2 \mu \mathrm{m}$ in diameter) and their concentration increases as $X_{\text {STDC }}$ increases toward equimolarity. For this composition, small crystallites could also be seen in the microscope (cf. large particle in the right-hand lower area of Fig. 2c), an observation that may indicate a slight rise in the Krafft temperature of the system, at higher amounts of bile salt. The coacervate consisting of $\mathrm{L}_{1}$ domains dispersed in $\mathrm{L}$ and formed around equimolarity also shows droplets in Brownian motion (Fig. 2d). It is likely that the nature of the milky dispersion is similar to the coacervate, except that for the
Fig. 2 Light micrographs for samples at constant total surfactant concentration $c_{\mathrm{t}}=20 \mathrm{mM}$ and increasing amount of bile salt: a $0.10 ;$ b 0.20 ; c $0.35 ;$ d 0.50 . $B a r=10 \mu \mathrm{m}$

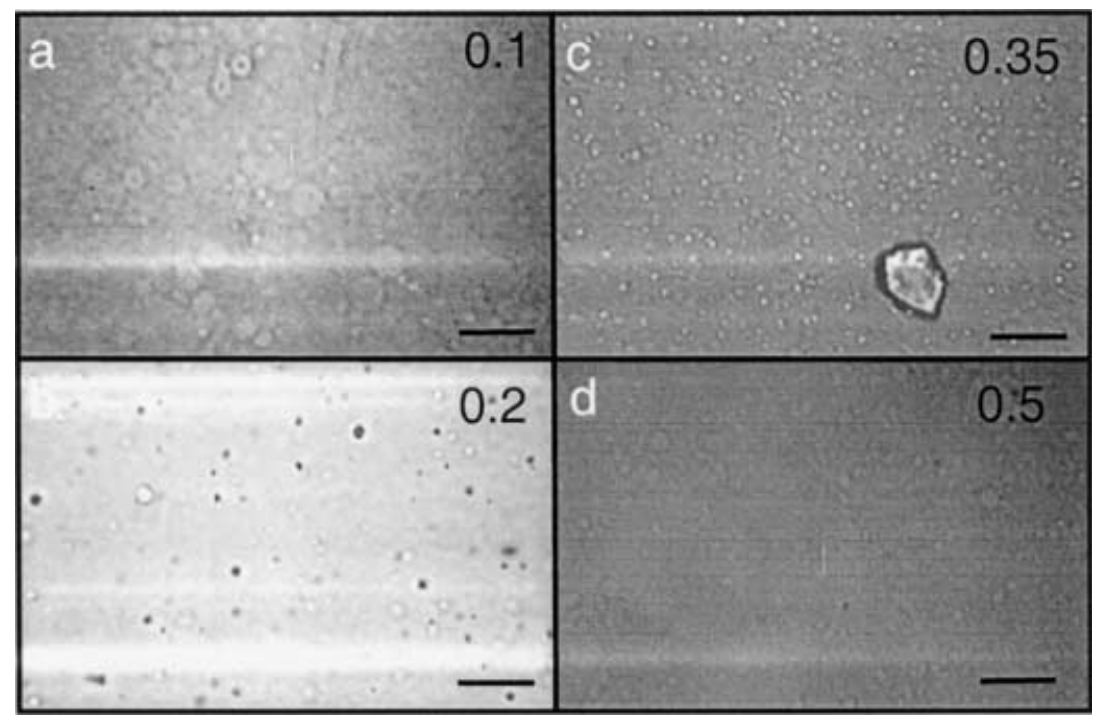


latter there is a macroscopic phase separation between $\mathrm{L}$ and $\mathrm{L}_{1}$, while in the former a fine dispersion is formed.

The characterization of the size and structure of the vesicles occurring in the bluish turbid samples was further carried out by cryo-TEM imaging, which probes optimally particle sizes between 10 and $500 \mathrm{~nm}$. Again the presence of round, integral vesicle structures was confirmed, as illustrated by the micrographs in Fig. 3 for samples at $c_{\mathrm{t}}=20 \mathrm{mM}$ and $X_{\mathrm{STDC}}=0.025,0.10$ and 0.15 , respectively. At $2.5 \mathrm{~mol} \%$, large unilamellar vesicles, with size ranges varying from 100 to $600 \mathrm{~nm}$ are seen throughout the sample (Fig. 3a). The majority of vesicles are in the range $200-500 \mathrm{~nm}$. With increasing concentration of STDC, at $10 \mathrm{~mol} \%$, some differences are apparent (Fig. 3b). First, the average size seems to have shifted somewhat to larger size, since most of the vesicles observed are in the range 400-700 nm; only a few vesicle below $400 \mathrm{~nm}$ are observed. Moreover, besides round vesicles other structures are observed; in particular it is possible to see long tubular bilayers linking different vesicular structures (marked i in Fig. 3b). The aggregate marked with ii is quite interesting, as it consists of an almost polyhedral vesicle with four long tubular "arms" possibly connecting other vesicular aggregates. This type of structure has not been seen before in neat DDAB solutions and dispersions and is clearly a structural effect resulting from the presence of the bile salt in the bilayer assembly. The aggregates seen in cryo-TEM at $15 \mathrm{~mol} \%$ STDC are shown in Fig. 3c. Here, one sees that the majority of vesicles are relatively small (20-200 nm) with a few large ones (up to $600 \mathrm{~nm}$ ). In addition it is striking to observe the occurrence of small spheroidal aggregates, which appear as darks spots, indicating a high and uniform mass thickness contrast. These particles have sizes of the order of 10-80 nm, with the majority in the range $10-40 \mathrm{~nm}$. It is evident that they are too large to be micellar aggregates; they probably consist of nanosized droplets, which at higher $X_{\text {STDC }}$ become large enough and thus visible in the light microscope.

\section{Effect of bile salt on the dilute lamellar phase}

A light microscopy study of more concentrated samples in the dilute region was also carried out, with the goal of probing the effect of bile salt on the structure and stability of the bilayer aggregates. The types of structures imaged in the polarizing microscope when bile salt is added to the $\mathrm{D}_{\mathrm{I}}$ lamellar phase, at $10 \mathrm{wt} \% \mathrm{DDAB}$, are shown in Fig. 4. At $0.5 \mathrm{wt} \%$ STDC, the large vesicular structures are still present in the biphasic lamellar dispersion as can be seen from the birefringent spherulites (Maltese crosses) in Fig. 4a. This optical effect arises when multilamellar vesicles (onions) in a dispersion are submitted to polarized light. However, at 1.0 $\mathrm{wt} \%$ STDC $\left(X_{\text {STDC }}=0.08\right)$, the structures present are

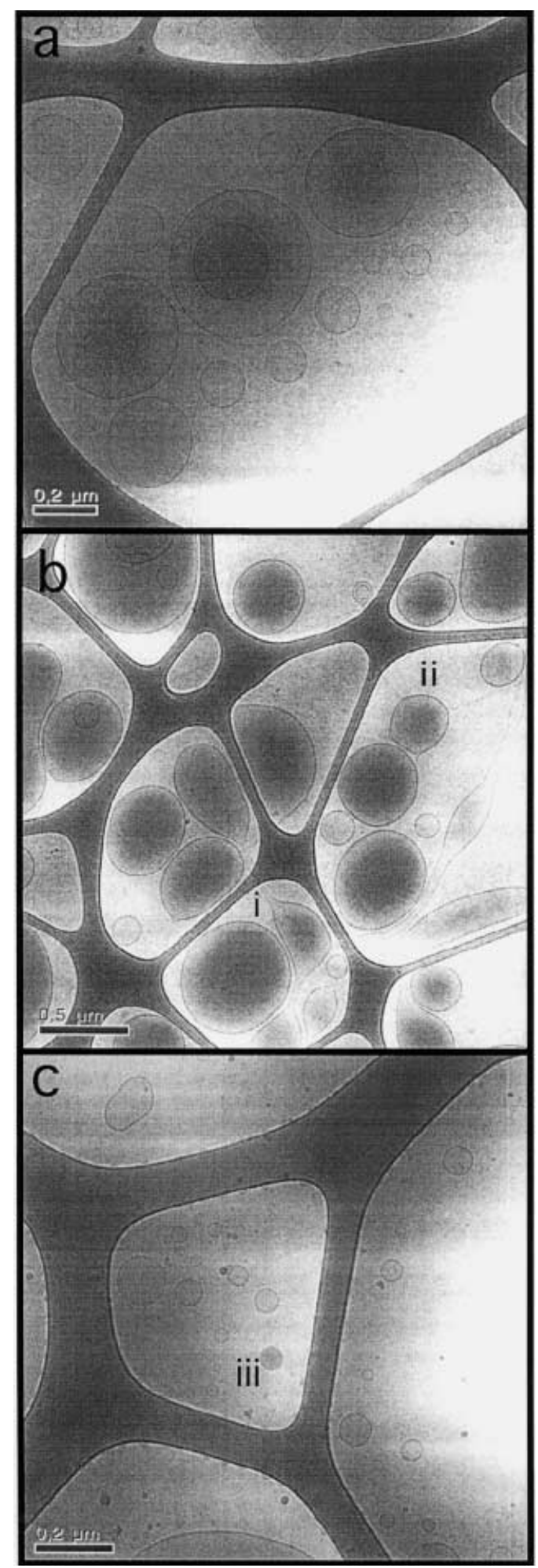

Fig. 3 Cryogenic transmission electron microscopy imaging of the bluish dispersions at total surfactant concentration $c_{\mathrm{t}}=20 \mathrm{mM}$ : a $X_{\mathrm{STDC}}=0.025 ; \mathbf{b} X_{\mathrm{STDC}}=0.10 ; \mathbf{c} X_{\mathrm{STDC}}=0.15$ 


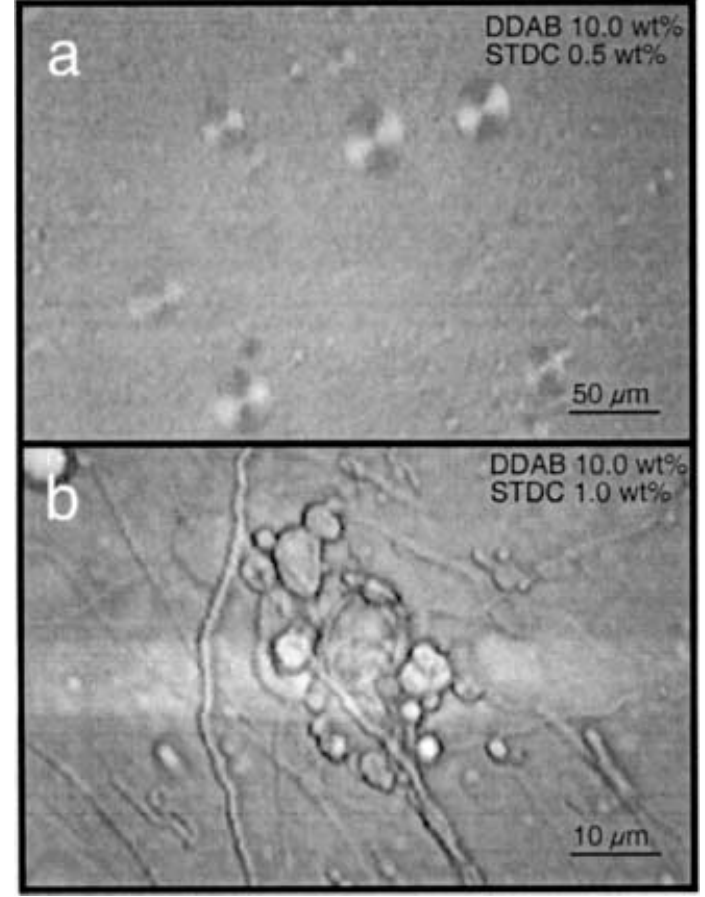

Fig. 4 The effect of the bile salt on the DDAB dilute lamellar phase, as imaged in the polarizing light microscope: a $10 \mathrm{wt} \% \mathrm{DDAB}$, $0.5 \mathrm{wt} \%$ STDC; b $10 \mathrm{wt} \%$ DDAB, $1.0 \mathrm{wt} \%$ STDC

very different. Long tubular structures are seen, looking very similar to fibers. The fiberlike aggregates are the dominant structures but it is possible to see also areas where vesicles have coalesced into big clusters, indicating the decreased colloidal stability of the bilayer aggregates (a salt-type effect). The dominant fibers bear some structural resemblance both to the microtubules imaged by cryo-TEM in Fig. $3 \mathrm{~b}$ and to the long rodlike structures formed by the bile salt in water at high concentrations, as imaged by light microscopy in recent work [46].

\section{Water self-diffusion NMR}

In order to complement the microscopy results, water self-diffusion measurements were performed for a series of samples at constant total surfactant concentration, $c_{\mathrm{t}}=20$ and $10 \mathrm{mM}$, and increasing values of $X_{\mathrm{STDC}}$ in the range $0-20$ (Fig. 5a, b, respectively). The profiles of the water ${ }^{1} \mathrm{H}$ echo decay for the vesicular dispersions seen in Fig. 5 are very similar to those which have been previously reported for catanionic DDAB-sodium dodecyl sulfate (SDS) vesicles (both cationic- and anionic-rich vesicles) $[24,25]$. A more detailed discussion of the theoretical aspects behind these experimental results for the solvent self-diffusion can be found in these latter reports.
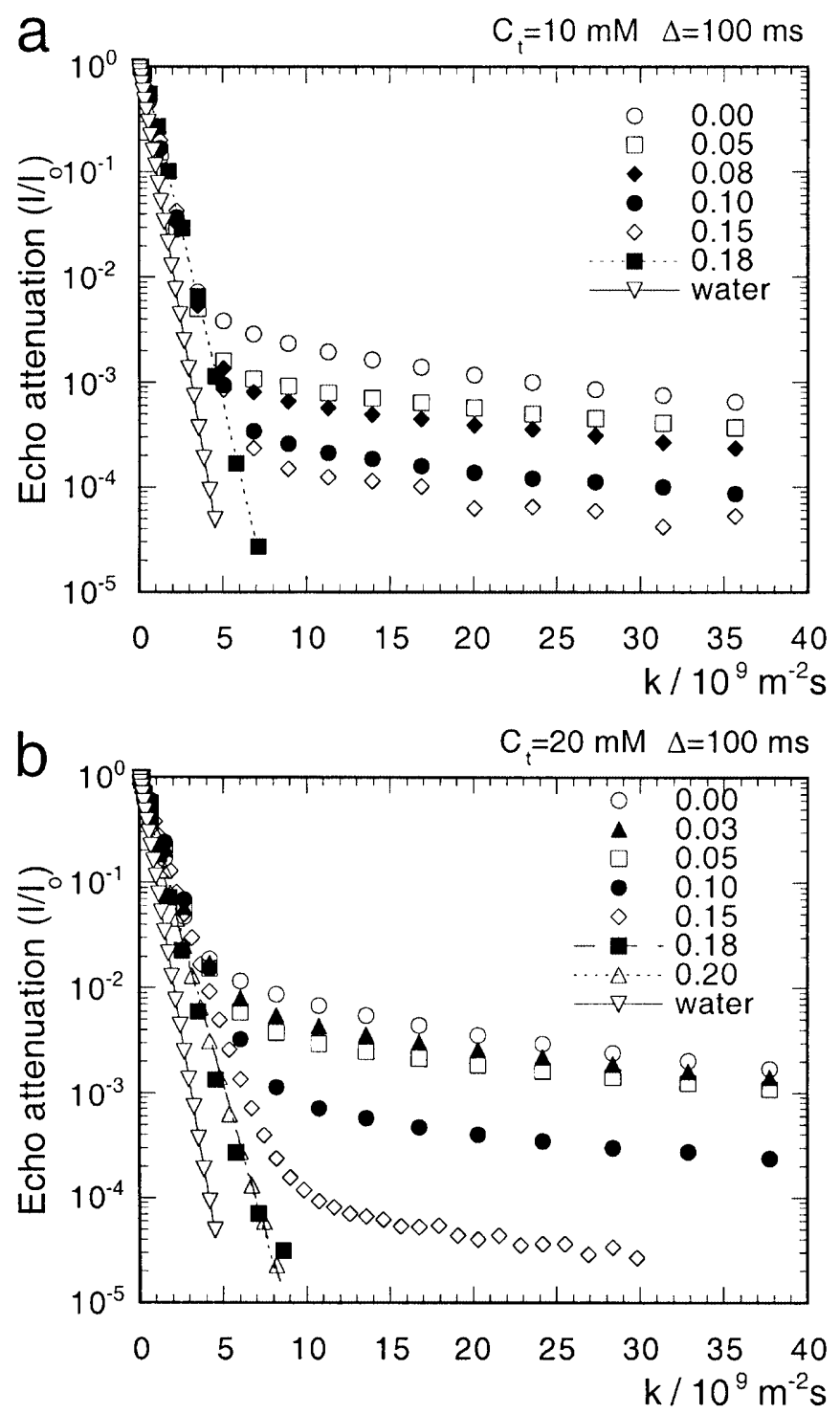

Fig. 5 Water NMR self-diffusion measurements for the DDABSTCD bluish dispersions at $25{ }^{\circ} \mathrm{C}$. The profile of the ${ }^{1} \mathrm{H}$ echo decays as a function of $X_{\mathrm{SDTC}}$, for a timescale $\Delta=100 \mathrm{~ms}$ is shown a for $c_{\mathrm{t}}=10 \mathrm{mM}$ and $\mathbf{b}$ for $c_{\mathrm{t}}=20 \mathrm{mM}$. The straight lines shown are monoexponential fits for the echo decay, (free diffusion)

Instead of a single linear decay, typical for free, unrestricted diffusion, a fast- and a slow-diffusing component are simultaneously observed in Fig. 5a and b. The slow component is nonmonoexponential and its fraction is very small (less than $1 \%$ ). Its profile represents a rather intricate convolution between size polydispersity and restricted diffusion and, as such, fitting procedures have not yielded meaningful data; however, they do allow a qualitative discussion. This slow component has been assigned to the presence of very large vesicular structures [24, 25, 47]. Large vesicles show a long residence time, $\tau_{\text {res }}$, for water and if $\tau_{\text {res }}$ is longer than 
the time scale of the NMR experiment, $\Delta$, the entrapment effect is captured in the NMR diffusion experiment. So, within the experimental timescale used $(100 \mathrm{~ms})$, the water inside these large vesicles feels entrapment and is under restricted diffusion, meaning that the echo profile is dependent on $\Delta$. For $\Delta=100 \mathrm{~ms}$, a typical root-meansquare displacement for water is of the order of $10 \mu \mathrm{m}$; the occurrence of a slow-diffusing component implies that there is a detectable fraction of vesicles with sizes above this value. The previous light microscopy observations confirm this observation.

All the samples in Fig. 5 show a complex decay, except at $X_{\mathrm{STDC}}=0.18$ and 0.20 , where single-exponential decays are observed (fitted straight lines). These simple decays indicate a situation of common free diffusion for water, consistent with the presence of small particles for such samples (as shown in Fig. 2b). If the particles consist of droplets then this result implies that water is in fast exchange between the two coexisting phases in the dispersion, i.e. on the experimental time scale chosen; no restricted diffusion is observed for the water molecules inside the droplets. It is also apparent in Fig. 5 that the fraction of the slow component decreases gradually upon increasing $X_{\text {STDC. }}$ In Fig. 5b, from 0 to 0.10 and from 0.10 to 0.15 , the decrease is roughly of 1 order of magnitude and is thus rather significant. Bearing in mind that the slow-diffusing component has been traced to very large vesicular structures (in slow exchange), a decrease in its fraction is simply assigned to a decrease in the amount of water in restricted diffusion inside the vesicles. If the vesicles grow between $X_{\mathrm{STDC}}=0$ and 0.10 , it is likely that the increase is high enough that some of the vesicles change from a situation of restricted to free diffusion for the entrapped water - thus their fraction no longer contributes to the slow-diffusing component.

If the slow-diffusing component is discarded, on account of its very small fraction, the fast-diffusing component, $\mathrm{D}_{\text {fast }}$, divided by the neat water coefficient $D_{0}\left(D / D_{\mathrm{o}}\right.$ is the reduced coefficient $)$, can be meaningfully plotted as a function of $X_{\text {STDC }}$ (Fig. 6). It is interesting to note that $D / D_{0}$ varies in a nonmonotonic fashion with increasing bile salt concentration, both at 10 and at $20 \mathrm{mM}$. The initial decrease in $D / D_{0}$ indicates a higher obstructing effect for the water molecules, an observation which is in agreement with the apparent increase in size detected by cryo-TEM. The subsequent increase in $D / D_{0}$, as $X_{\text {STDC }}$ increases from 0.10 to 0.15 , may reflect the fact that aggregates other than vesicles are present, having a lower obstructing volume. Indeed the small particles seen by cryo-TEM (Fig. 3c) are consistent with this effect. Further analysis of the $D / D_{0}$ values can yield a rough estimation of the size of the vesicles. Assuming that the vesicles behave as relatively large, monodisperse, hard spheres and that a situation of fast exchange is present, the vesicle volume fraction, $\Phi_{\text {ves }}$, is obtained from $D / D_{0}$ through

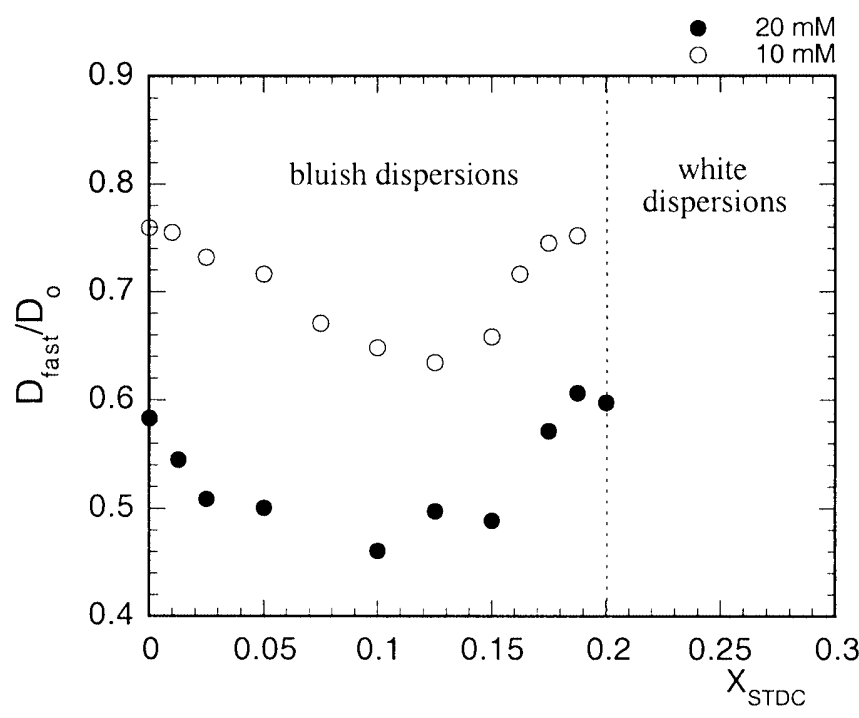

Fig. 6 The reduced diffusion coefficient of water, $D / D_{0}$, where $D$ is observed self-diffusion coefficient and $D_{0}$ is the self-diffusion coefficient for neat water at $25^{\circ} \mathrm{C}$, as a function of $X_{\text {STDC }}$, for series at $c_{\mathrm{t}}=10 \mathrm{mM}$ and $c_{\mathrm{t}}=20 \mathrm{mM}$

$\Phi_{\text {ves }}=2\left(1-D / D_{0}\right) /\left(2+D / D_{0}\right)$

and an estimation of the vesicle radius can then be obtained through

$R_{\mathrm{ves}}=6 \Phi_{\mathrm{ves}} / N_{\mathrm{A}} c_{\mathrm{t}} a_{\mathrm{s}}$

where $N_{\mathrm{A}}$ is the Avogadro number, $c_{\mathrm{t}}$ is the total surfactant molar concentration and $a_{\mathrm{s}}$ is the average surfactant headgroup area. An estimation of the vesicle volume fraction and radius is given in Table 1 , for samples at $c_{\mathrm{t}}=20 \mathrm{mM}$ and $X_{\mathrm{STDC}}=0-0.10$ (where only vesicles were imaged by microscopy methods). For the neat DDAB dispersion, a value of $a_{\mathrm{s}}=65 \AA^{2}$ was used [48], whereas for the mixtures $\mathrm{a}_{\mathrm{s}} \approx 67 \AA^{2}$ was employed. The latter value assumes that

1. The bile salt molecule is in the mixed bilayer with a position normal to the polar-apolar interface (instead of the parallel).

2. The molecule occupies at most a cross-sectional area of $113 \AA^{2}$ (determined by the cross-sectional area of the ring system [2]).

Table 1 Estimated vesicle volume fraction and vesicle radius from the reduced water self-diffusion coefficients for didodecyldimethylammonium bromide-sodium taurodeoxycholate (STDC) bluish dispersions at $c_{\mathrm{t}}=20 \mathrm{mM}, 25^{\circ} \mathrm{C}$

\begin{tabular}{llll}
\hline$X_{\text {STDC }}$ & $D / D_{0}$ & $\Phi_{\text {ves }}$ & $R_{\text {ves }}(\mathrm{nm})$ \\
\hline 0 & 0.58 & 0.32 & 247 \\
0.013 & 0.55 & 0.36 & 266 \\
0.025 & 0.51 & 0.39 & 291 \\
0.050 & 0.50 & 0.40 & 297 \\
0.100 & 0.46 & 0.44 & 326 \\
\hline
\end{tabular}


3. The resulting effect on the average $a_{\mathrm{s}}$ is a balance between the presence of the bulkier STDC molecule in the bilayer (on a molecular ratio determined by $\left.X_{\text {STDC }}\right)$ and the DDAB-bile salt headgroup attractions.

In view of the number of assumptions made (some of which are relatively crude) the values are to be taken on a semiquantitative basis. The high volume fractions of the vesicles in Table $1(0.3-0.4)$ are nevertheless fairly consistent with the high concentration of the vesicles imaged by light microscopy and the turbidity of the samples. The magnitude and the increase of the average vesicle diameter from roughly 500 to $600 \mathrm{~nm}$, with increasing bile salt content, are also in agreement with the observations done by cryo-TEM.

Comparison with other mixed systems

Cryo-TEM and light microscopy have shown that DDAB vesicles, in the dilute region (less than $1 \mathrm{wt} \%$ DDAB), are able to incorporate a maximum amount of bile salt of about $20 \mathrm{~mol} \%$. The mixed DDAB-bile salt vesicles are spherical, large and unilamellar, and their dispersions present a long-term stability that may indicate the presence of true equilibrium structures, at least for some compositions of the mixture. Thermodynamic stability, rather than the more common kinetic one usually observed for lipid vesicles, has been verified in other catanionic vesicles $[19,20,21,24,25]$. The increase in vesicle size as the bile salt is incorporated in the bilayer, up to some concentration, is probably an effect just due to the partial charge neutralization of the bilayer (inducing a lower mean curvature) by the anionic surfactant. This effect can be considered as nonspecific, since, for example, in the DDAB-SDS system a similar effect was reported [25]. However, the rigid ring-based structure of the bile salt with its $\mathrm{OH}$ groups seems to bring some peculiar features of aggregation, such as the formation of long tubular structures, which clearly deserve further investigation.

The type of effects observed in this catanionic system, upon bile salt addition, show some differences from those observed for the phospholipid-bile salt systems. We note that the studies on the latter systems are aimed, in general, at the disintegration process of lipid vesicles by bile salts, implying that some of the structures observed might only be metastable and are not the true equilibrium ones. It has been shown that when added in subequimolar amounts, sodium cholate causes an increase in the size of the small unilamellar lecithin vesicles (prepared by sonication) and an opening of the vesicles [5]. Closer to equimolar ratios, there is the formation of bilayer patches, followed by mixed cylindrical micelles (roughly at equimolarity) and, eventually, small globular micelles. In the DDAB-STDC system, phase separation (formation of fine dispersions) occurs instead and well before the equimolar ratio $\left(X_{\mathrm{STDC}}>0.20\right)$, indicating how the much stronger associative behavior (than in the lipid-bile salt case) brings about a significant change of the phase behavior.

\section{Conclusion}

The phase behavior for the very dilute DDAB-rich area of the DDAB-STDC-water system has been investigated at $25^{\circ} \mathrm{C}$, denoting the occurrence of bluish bilayer dispersions, whitish fine dispersions and a clear liquid two-phase region in the close vicinity of equimolar compositions. The vesicular dispersions formed by the double-chained amphiphile alone in water are able to incorporate some bile salt (roughly $20 \mathrm{~mol} \%$ ), showing large, integral and polydisperse unilamellar vesicles, $100 \mathrm{~nm}$ to several microns in size. Cryo-TEM and self-diffusion results indicate that the average aggregate size increases with the concentration of bile salt on the membrane, corresponding approximately to $10 \mathrm{~mol} \%$ at $c_{\mathrm{t}}=20 \mathrm{mM}$. The formation of long tubular structures connecting vesicles and small spheroidal dropletlike aggregates has also been imaged by microscopy.

Acknowledgements We are grateful to C. La Mesa and O. Regev for fruitful discussions. E.F.M. acknowledges support from TFR (Sweden) and C.I.Q.(U.P.), linha 5, Fundação para a Ciência e Tecnologia (Portugal).

\section{References}

1. Carey MC, Small DM (1970) Am J Med 49:590

2. Small DM (1971) In: Nair PP, Kritchevsky D (eds) The bile acids, vol 1. Plenum, New York, p 249

3. Kaplun A, Talmon Y, Konikoff FM, Rubin M, Eitan A, Tadmor M, Lichtenberg D (1994) FEBS Lett 340:78
4. Ulmius $\mathbf{J}$, Lindblom $\mathrm{G}$, Wennerström $\mathrm{H}$, Johansson LB-A, Fontell K, Söderman O, Arvidson G (1982) Biochemistry 21:1553

5. Walter A, Vinson PK, Kaplon A, Talmon Y (1991) Biophys J 60:1315

6. Hjelm RP, Thiyagarajan P, AlkanOnyuksel H (1992) J. Phys Chem 96:8653
7. Pedersen JS, Egelhaaf SU, Schurtenberger P (1995) J Phys Chem 99:1299

8. Cohen DE, Thurston GM, Chamberlin RA, Benedek GB, Carey MC (1998) Biochemistry 37:14798

9. Luk AS, Kaler EW, Lee SP (1997) Biochemistry 36:5633 
10. Svärd M, Schurtenberger P, Fontell K, Jönsson B, Lindman B (1988) J Phys Chem 92:2261

11. Edwards K, Almgren M (1992) Langmuir 8:824

12. Li C-Y, Wiedmann TS (1996) J Phys Chem 100:18464

13. Mesa CL, Khan A, Fontell K, Lindman B (1985) J Colloid Interface Sci 103:373

14. Swanson-Vethamuthu M, Almgren M, Bergenståhl B, Mukhtar E (1996) J Colloid Interface Sci 178:538

15. Khan A, Marques E (1997) In: Robb ID (ed) Specialist surfactants. Blackie, London, pp 37-76

16. Kronberg B (1997) Curr Opin Colloid Interface Sci 2:456

17. Ogino K, Masahiko A (eds) (1993) Mixed surfactant systems. Dekker, New York

18. Holland PM, Rubingh DN (eds) (1992) Mixed surfactant systems. American Chemical Society, Washington, DC

19. Kaler EW, Murthy AK, Rodriguez BE, Zasdzinski JAN (1989) Science 245:1371

20. Kaler EW, Herrington KL, Murthy AK, Zasadzinski JA (1992) J Phys Chem 96:6698-6707

21. Ambühl M, Bangerter E, Luisi PL, Skrabal P, Watzke HJ (1993) Prog Colloid Polym Sci 93:183
22. Filipovic-Vincekovic N, Bujan M, Dragcevic D, Nekic N (1995) Colloid Polym Sci 273:182-188

23. Regev O, Khan A (1996) J Colloid Interface Sci 182:95

24. Marques EF, Regev O, Khan A, Miguel MG, Lindman B (1998) J Phys Chem B 102:6746

25. Marques EF, Regev O, Khan A, Miguel MG, Lindman B (1999) J Phys Chem 103:8353

26. Marques E, Khan A, Miguel MG, Lindman B (1993) J Phys Chem 97:4729

27. Caria A, Khan A (1996) Langmuir 12:6282

28. Edlund H, Sadaghiani A, Khan A (1997) Langmuir 13:4953

29. Fontell K, Ceglie A, Lindman B, Ninham B (1986) Acta Chem Scand A 40:247

30. Warr GG, Sen R, Evans DF, Trend JE (1988) J Phys Chem 92:774

31. Dubois M, Zemb T (1991) Langmuir 7:1352

32. Zemb T, Gazeau D, Dubois M, GulikKrzywicki T (1993) Europhys Lett 21:759

33. Miller DD, Bellare JR, Evans DF, Talmon Y, Ninham BW (1987) J Phys Chem 91:674

34. Dubois M, Gulik-Krzywicki T, Cabane B (1993) Langmuir 9:673
35. Regev O, Khan A (1994) Prog Colloid Polym Sci 97:298

36. Svitova TF, Smirnova YP, Pisarev SA, Berezina NA (1995) Colloids Surf A 101:107

37. Marques EF, Regev O, Edlund H, Khan A (2000) Langmuir 16:8255

38. Van de Pas JC (1991) Tenside Surfactants Deterg 28:158

39. Lindman B, Söderman O, Wennerström H (1987) In: Zana R (ed) Surfactant solutions, new methods of investigation. Dekker, New York, p 295

40. Söderman O, Stilbs P (1994) Prog Nucl Magn Reson Spectrosc 26:445

41. Stilbs P (1987) Prog Nucl Magn Reson Spectrosc 19:1

42. Price WS (1997) Concepts Magn Reson 9:299

43. Kachar B, Evans DF, Ninham BW (1984) J Colloid Interface Sci 100:287

44. Dubois M, Cabane B (1994) Langmuir $10: 1615$

45. Caboi F, Monduzzi M (1996) Langmuir 12:3548

46. Marques EF, Edlund H, Mesa CL, Khan A (2000) Langmuir 16:5178

47. Marques EF (2000) Langmuir 16:4798

48. Zemb T, Belloni L, Dubois M, Marcelja S (1992) Prog Colloid Polym Sci $89: 33$ 